\title{
Chinese-English Semantic Resource Construction
}

\author{
Bonnie J. Dorr ${ }^{\dagger}$, Gina-Anne Levow ${ }^{\dagger}$, Dekang Lin ${ }^{\ddagger}$, Scott Thomas ${ }^{\dagger}$ \\ ${ }^{\dagger}$ Institute for Advanced Computer Studies \\ University of Maryland \\ \{bonnie,gina,scthmas\}@umiacs.umd.edu \\ $\ddagger$ Department of Computing Science \\ University of Alberta \\ Edmonton, Alberta, Canada, T6G 2H1 \\ lindek@cs.ualberta.ca
}

\begin{abstract}
We describe an approach to large-scale construction of a semantic lexicon for Chinese verbs. We leverage off of three existing resourcesa classification of English verbs called EVCA (English Verbs Classes and Alternations) (Levin, 1993), a Chinese conceptual database called HowNet (Zhendong, 1988c; Zhendong, 1988b; Zhendong, 1988a) (http://www.how-net.com), and a large machine-readable dictionary called Optilex. The resulting lexicon is used for determining appropriate word senses in applications such as machine translation and cross-language information retrieval.
\end{abstract}

\section{Introduction}

With the growing quantity of online multilingual information, automatic and semi-automatic techniques for lexical acquisition are more critical now than ever before. We describe an approach to large-scale construction of a semantic lexicon for Chinese verbs. We leverage off of three existing resources - a classification of English verbs called EVCA (English Verbs Classes and Alternations) (Levin, 1993), a Chinese conceptual database called HowNet (Zhendong, 1988c; Zhendong, 1988b; Zhendong, 1988a) (http://www.how-net.com), and a large machine readable Chinese-English dictionary called Optilex. ${ }^{1}$

Our approach involves extraction of candidate translations from Optilex for each of the Chinese verbs occurring in HowNet. We then create links between Chinese concepts and English classes using thematic-role mappings between HowNet entries and EVCA-based entries. Each ChineseEnglish link is subsequently associated with a sense from WordNet (Miller and Fellbaum, 1991), thus producing a new Asian companion to the current (Euro)WordNet initiative. The resulting lexicons are used for determining appropriate word senses in applications such as machine translation and cross-language information retrieval.

Several researchers have investigated the problem of assigning class-based senses to verbs (Dang et al., 1998), (Dorr and Jones, 1999), (Dorr and Jones, 1996) (Dorr, 1997), (Jones et al., 1994), (Nomura et al., 1994) (Olsen et al., 1998), (Palmer and Wu, 1995), (Palmer and Rosenzweig, 1996), and (Saint-Dizier, 1996). This work extends the techniques described by (Palmer and Wu, 1995), which used a concept space to produce a hierarchical organization of Chinese verbs. The extensions include the use of the entire EVCA database rather than a small set of verbs (the break class) and the provision of a thematic-role based filter. We adopt a technique that is similar in flavor to the

\footnotetext{
${ }^{1}$ Optilex is the machine-readable version of the CETA dictionary, licensed from the MRM corporation, Kensington, MD.
}

intersective-class approach of (Dang et al., 1998), with the following extensions: (1) Concept alignment across two different language hierarchies (Chinese and English) rather than one; (2) Mappings between Chinese and English thematic roles; and (3) Hooks into WordNet senses for both languages.

The next section describes the HowNet conceptual database. Following these, we will describe the approach we used to produce the concept-to-class correspondence. Section 4. presents the result of our automatic acquisition experiment.

\section{HowNet Conceptual Database}

HowNet is an on-line conceptual common-sense knowledge base that contains hierarchical information relating concepts to the associated Chinese word. Our focus is on the verb hierarchy, which has the structure shown in Table 1.

The number labels given here are our own; we use these for indicating the level of each concept in the HowNet database. Note that the highest two concepts in the verb hierarchy are "static" (V.1) and "act" (V.2). These correspond, respectively, to verbs such as 成为 (become under the "static" node V.1.1.1) and 开始 (start under the "act" node V.2.1.1). The levels go much deeper than these, with the lowest ones at 8 levels deep, e.g., V.1.2.1.6.3.3.1.15 itch.

Within each of the HowNet classes is a thematic-role specification. For example, the verb "cure" has the thematic-role specification (agent, patient, content, tool). Consider the sentence The doctor cured the man of pneumonia using antibiotics. The roles in the specification have the following binding, respectively, for this sentence : doctor, man, pneumonia, antibiotics. ${ }^{2}$ The thematic-role specifications are used for prioritizing candidate HowNet-EVCA associations, as will be described below.

\footnotetext{
${ }^{2}$ Thematic-role specifications and their use in generation of natural-language translations are described further in (Dorr et al., 1998).
} 


V.1 |static|
V.1.1 |relation|
V.1.1.1 |isa|
V.1.1.2 | possession|
V.1.1.3 |comparison|
V.1.1.4 | suit|
V.1.1.5 |inclusive|
V.1.1.6 |connective|
V.1.1.7 |CauseResult|
V.1.1.8 |TimeOrSpace|
V.1.1.9 |arithmetic|
V.1.2 |state|
V.1.2.1 |StatePhysical|
V.1.2.2 |StateMental|

\author{
V.2 |act| \\ V.2.1 ActGeneral| \\ V.2.1.1 |start| \\ V.2.1.2 |do| \\ V.2.1.3 |DoNot| \\ V.2.1.4 |Cease| \\ V.2.1.5 |Wait| \\ V.2.2 |ActSpecific| \\ V.2.2.1 |AlterGeneral| \\ V.2.2.2 AlterSpecific| \\ V.2.3 |AlterRelation| \\ V.2.3.1 |AlterIsa| \\ V.2.3.2 |AlterPossession| \\ V.2.3.3 AlterComparison| \\ V.2.3.4 |AlterFitness| \\ V.2.3.5 |AlterInclusion| \\ V.2.3.6 |AlterConnection| \\ V.2.3.7 |AlterCauseResult| \\ V.2.3.8 AlterLocation| \\ V.2.3.9 |AlterTimePosition|
}

V.2.4 |AlterState|

V.2.4.1 |AlterPhysical|

V.2.4.2 |AlterStateNormal|

V.2.4.3 |AlterStateGood|

V.2.4.4 |AlterQuantity|

V.2.4.5 |AlterStateBad|

V.2.4.6 |AlterMental|

V.2.5 |AlterAttribute|:

V.2.5.1 |MakeHigher|

V.2.5.2 |MakeLower|

V.2.5.3 |AlterAppearance|

V.2.5.4 |AlterMeasurement|

V.2.5.5 |AlterProperty|

V.2.6 |MakeAct|:

V.2.6.1 |CauseToDo|

V.2.6.2 |CauseNotToDo|

V.2.6.3 |use|

Table 1: HowNet Verb Hierarchy

\section{Approach}

We have associated 478 Chinese HowNet concepts with 485 EVCA classes, demonstrating a clear concept-to-class correspondence in a large majority of the cases. ${ }^{3}$ The mapping between Chinese HowNet and English EVCA (hence WordNet) involves three steps.

The first step is to produce all possible English Optilex glosses (translations) for all 12342 Chinese verbs in HowNet and associate each Chinese verb with one or more of the 478 HowNet concepts, forming 48,884 verb-toconcept candidates. For example, there is a common Chinese verb 拉 (la) that is multiply ambiguous, corresponding to 13 Optilex-based English glosses: slash, cut, chat, pull, drag, transport, move, raise, help, implicate, involve, defecate, and pressgang. This verb is associated with 9 HowNet concepts: |Transport|, |Attract|, |Excrete|, |Force|, |Help|, |Include|, |Pull|, |Recreation|, and |Talk|.

The second step involves associating each verb-toconcept candidate with one or more of the 485 EVCA classes, forming an average of 2 thousand verb-to-class entries per HowNet concept (on the order of 1 million verb-to-class candidates, total). For example, the Chinese verb 拉 (la) is associated with 22 EVCA classes: Admire (31.2.b, implicate, involve); Amuse (31.1.b, transport, move, cut; Braid (41.2.2, cut); Breathe (40.1.2, defecate); Build (26.1.a, cut); Carry (11.4.i, carry, pull, drag); Chitchat (37.6.a, chat); Crane (40.3.2, raise); Cut (21.1.a, slash, cut); Cut (21.1.d, cut); Equip (13.4.2, help); Force (12.a.ii, pull); Get (13.5.1.a, pull); Grow (26.2.a.ii, raise); Hurt (40.8.3, pull, cut); Meander (47.7.a, cut); Play (009, pawn); Put (9.4.a, raise); Search (35.2.a, drag); Send (11.1, smuggle, transport, ship, convey); Send Slide(11.2.b,

\footnotetext{
${ }^{3}$ There are actually more than 800 concepts in HowNet that define events. The number was reduced to 478 for the purpose of this preliminary experiment; a more in-depth acquisition process is currently underway to fill out the final $300+$ concepts. See (Dorr et al., 2000).
}

move); Split (23.2.b, cut, pull).

The final step is to partition each HowNet concept into groups of Chinese-English pairs whose English glosses correspond to EVCA classes. This involves three subtasks:

- Order the candidate EVCA classes so that the highestranking classes are those that contain the highest number of English verbs matching the Optilex glosses.

- In cases where a tie-breaker is needed, reorder the candidate EVCA classes according to the degree to which the thematic-role specification in HowNet concept matches that of EVCA class.

- For each Chinese-English entry associated with the HowNet concept, assign the highest ranking candidate EVCA class.

Consider two HowNet concepts associated with the the Chinese verb 拉 (la): |Help| and |Transport|. The thematic-role specification associated with $|\mathrm{Help}|$ is (agent, patient, scope) (as in John helped him with his work). This specification most closely matches that of Equip EVCA Class (where 拉 (la) is translated as help) which has the specification _ag_th, mod-poss (with); thus, the $\mid$ Help $\mid$ HowNet concept is associated with the Equip EVCA Class, and the mapping between the two is (agent->ag), (patient->th), (scope->mod-poss).

On the other hand, the |Transport| HowNet concept is associated with the thematic-role specification (agent, patient, LocationIni, LocationFin, direction) (as in John transported the goods from Boston to New York (westward)). This specification most closely matches that of the Send EVCA Class (where 拉 (la) is translated as transport); thus, the |Transport|

Hownet concept is associated with the Send EVCA class, and the mapping between the two is (agent->ag), 


\begin{tabular}{|l|l|l|l|l|l|l|}
\hline Number of EVCA Classes per Concept: & 0 & 1 & 2 & 3 & 4 & 5 \\
\hline Number of HowNet Concepts: & 2 & 371 & 71 & 20 & 10 & 4 \\
\hline
\end{tabular}

Table 2: Partitioning of HowNet Concepts into EVCA Classes

\begin{tabular}{|l|l|}
\hline HowNet Concept & EVCA Class(es) \\
\hline $\mid$ Transport $\mid$ & 11.1 Send \\
\hline $\mid$ Help $\mid$ & 13.4.2 Equip \\
\hline $\mid$ Apologize $\mid$ & 32.2.a Long \\
\hline $\mid$ Naming $\mid$ & 29.3 Dub \\
\hline $\mid$ Judge $\mid$ & 29.4 Declare \\
\hline $\mid$ Moisten $\mid$ & 45.4.a Change of State \\
\hline $\mid$ Excrete $\mid$ & 40.1.2 Breathe \\
\hline $\mid$ TakeVehicle $\mid$ & 51.4.2.a.ii Motion by Vehicle \\
\hline $\mid$ PlayDown $\mid$ & 33.b Judgment (75\%), 31.2.a Admire (25\%) \\
\hline $\mid$ Establish $\mid$ & 29.2.c Characterize (90\%), 26.4.a Create (19\%) \\
\hline $\mid$ Decorate $\mid$ & 9.8.b Fill (50\%), 26.1.b Build (43\%), 9.9.ii Butter (25\%) \\
\hline $\mid$ Buy $\mid$ & 10.5 Steal (08\%), 13.5.1.a Get (30\%), 13.5.1.b.ii Get (54\%), 13.5.2.d Get (46\%) \\
\hline $\mid$ Teach $\mid$ & 29.2.c Characterize (24\%), 33.b Judgment (71\%), 37.9.a Advise (29\%), 37.1.a Transfer \\
& Message (45\%), 31.1.a Amuse (19\%) \\
\hline
\end{tabular}

Table 3: Examples of HowNet Partitionings with Respect to EVCA

(patient->th), (LocationIni->src),
(LocationFin->goal).

The end result is that the English glosses associated with 拉 (la) are filtered down to help in the Equip semantic class and transport in the Send semantic class; the corresponding WordNet senses are assigned (for free) from the handtagged EVCA database. These are Senses 1-3 in the case of transport (i.e., move/carry/displace) and Sense 1 in the case of help (i.e., aid/assist):

\section{- transport:}

Sense 1: transport

Sense 2: transport, carry

Sense 3: transport, send, ship

- help:

Sense 1: help, assist, aid

\section{Results}

Table 2 characterizes the number of EVCA classes required for coverage of 478 HowNet concepts. We consider the approach to be a success for several reasons: (1) Association of a unique EVCA class to a HowNet concept was achieved in 371 cases-77\% of the HowNet classes; (2) Most of the other cases partitioned the HowNet entries into 2 EVCA classes; (3) Only 2 cases did not correspond to any EVCA class (i.e., every word associated with the concept belonged to a different EVCA class); (4) There were no partitionings exceeding 5 EVCA classes.

Examples of the HowNet partitionings into EVCA classes are given in Table 3, with a focus on the cases where 1 partition was found. In cases where there is more than 1 partition, percentages are given with respect to the number of Chinese verbs in each HowNet class. ${ }^{4}$

\footnotetext{
${ }^{4}$ The astute reader will notice the percentages don't always to-
}

\section{Summary}

We have presented an approach to aligning two largescale online resources, HowNet and EVCA. The lexicon resulting from this approach is large-scale, containing 17284 Chinese-English conceptual links. The technique for producing these links involves matching semantic-role specifications in HowNet with those in EVCA. Our results indicate that the correspondence is very high between the 478 Chinese HowNet concepts and the 485 EVCA classes. Because each Chinese-English link is additionally associated with a WordNet sense, we see this resource as the first step toward producing a new Asian language companion to ongoing (Euro)WordNet initiatives.

\section{Acknowledgements}

The University of Maryland authors are supported, in part, by PFF/PECASE Award IRI-9629108, DOD Contract MDA904-96-C-1250, and DARPA/ITO Contract N6600197-C-8540. Dekang Lin is supported by Natural Sciences and Engineering Research Council of Canada grant OGP121338.

\section{References}

Dang, Hoa Trang, Karin Kipper, Martha Palmer, and Joseph Rosenzweig, 1998. Investigating Regular Sense Extensions Based on Intersective Levin. In ACL/COLING 98, Proceedings of the 36th Annual Meeting of the Association for Computational Linguistics (joint with the 17th International Conference on Computational Linguistics). Montreal, Canada.

tal $100 \%$. This is because certain of the Chinese verbs are assigned to two different "partitionings." The resulting groups are, thus, not true partitions in the mathematical sense since they are not necessarily mutually exclusive. In the cases where the percentages total $100 \%$, the resulting groups are mutually exclusive. 
Dorr, Bonnie J., 1997. Large-Scale Acquisition of LCSBased Lexicons for Foreign Language Tutoring. In Proceedings of the ACL Fifth Conference on Applied Natural Language Processing (ANLP). Washington, DC.

Dorr, Bonnie J., Nizar Habash, and David Traum, 1998. A Thematic Hierarchy for Efficient Generation from Lexical-Conceptal Structure. In Proceedings of the Third Conference of the Association for Machine Translation in the Americas, AMTA-98, in Lecture Notes in Artificial Intelligence, 1529. Langhorne, PA.

Dorr, Bonnie J. and Douglas Jones, 1996. Acquisition of Semantic Lexicons: Using Word Sense Disambiguation to Improve Precision. In Proceedings of the Workshop on Breadth and Depth of Semantic Lexicons, 34th Annual Conference of the Association for Computational Linguistics. Santa Cruz, CA.

Dorr, Bonnie J. and Douglas Jones, 1999. Acquisition of semantic lexicons: Using word sense disambiguation to improve precision. In Evelyne Viegas (ed.), Breadth and Depth of Semantic Lexicons. Norwell, MA: Kluwer Academic Publishers.

Dorr, Bonnie J., Gina-Anne Levow, Dekang Lin, and Scott Thomas, 2000. Large-Scale Construction of ChineseEnglish Semantic Hierarchy. Technical Report LAMP TR 040, UMIACS TR 2000-17, CS TR 4120, University of Maryland, College Park, MD.

Jones, Douglas, Robert Berwick, Franklin Cho, Zeeshan Khan, Karen Kohl, Naoyuki Nomura, Anand Radhakrishnan, Ulrich Sauerland, and Brian Ulicny, 1994. Verb Classes and Alternations in Bangla, German, English, and Korean. Technical report, Massachusetts Institute of Technology.

Levin, Beth, 1993. English Verb Classes and Alternations: A Preliminary Investigation. Chicago, IL: University of Chicago Press.

Miller, George A. and Christiane Fellbaum, 1991. Semantic Networks of English. In Beth Levin and Steven Pinker (eds.), Lexical and Conceptual Semantics, Cognition Special Issue. Amsterdam, The Netherlands: Elsevier Science Publishers, B.V., pages 197-229.

Nomura, Naoyuki, Douglas A. Jones, and Robert C. Berwick, 1994. An architecture for a universal lexicon: A case study on shared syntactic information in Japanese, Hindi, Ben Gali, Greek, and English. In Proceedings of COLING-94. Kyoto, Japan.

Olsen, Mari Broman, Bonnie J. Dorr, and Scott C. Thomas, 1998. Enhancing Automatic Acquisition of Thematic Structure in a Large-Scale Lexicon for Mandarin Chinese. In Proceedings of the Third Conference of the Association for Machine Translation in the Americas, AMTA98, in Lecture Notes in Artificial Intelligence, 1529. Langhorne, PA.

Palmer, Martha and Joseph Rosenzweig, 1996. Capturing motion verb generalizations with synchronous tags. In Proceedings of the Second Conference of the Association for Machine Translation in the Americas. Montreal, Quebec, Canada.

Palmer, Martha and Zhibao Wu, 1995. Verb Semantics for English-Chinese Translation. Machine Translation,
10(1-2):59-92.

Saint-Dizier, Patrick, 1996. Semantic Verb Classes Based on 'Alternations' and on WordNet-like Semantic Criteria: A Powerful Convergence. In Proceedings of the Workshop on Predicative Forms in Natural Language and Lexical Knowledge Bases. Toulouse, France.

Zhendong, Dong, 1988a. Enlightment and Challenge of Machine Translation. Shanghai Journal of Translators for Science and Technology, 1:9-15.

Zhendong, Dong, 1988b. Knowledge Description: What, How and Who? In Proceedings of International Symposium on Electronic Dictionary. Tokyo, Japan.

Zhendong, Dong, 1988c. MT Research in China. In Proceedings of International Conference on New Directions in Machine Translation. Budapest. Also in New Directions in Machine Translation, 4 Distributed Language Translation edited by Dan Maxwell, Klaus Schubert and Toon Witkam, Foris Publications, Dordrecht. 